\title{
Classification of Brain Tumor using Convolutional Neural Networks
}

\author{
Shravya Shetty, Jyothi Shetty
}

\begin{abstract}
In medical science, brain tumor is the most common and aggressive disease and is known to be risk factors that have been confirmed by research. A brain tumor is the anomalous development of cell inside the brain. One conventional strategy to separate brain tumors is by reviewing the MRI pictures of the patient's mind. In this paper, we have designed a Convolutional Neural Network (CNN) to perceive whether the image contains tumor or not. We have designed 5 different $C N N$ and examined each design on the basis of convolution layers, max-pooling, and flattening layers and activation functions. In each design we have made some changes on layers i.e. using different pooling layers in design 2 and 4, using different activation functions in design 2 and 3, and adding more Fully Connected layers in design 5. We examine their results and compare it with other designs. After comparing their results we find a best design out of 5 based on their accuracy. Utilizing our Convolutional neural network, we could accomplish a training accuracy and validation accuracy of design 3 at 100 epochs is $99.99 \%$ and $92.34 \%$, best case scenario.
\end{abstract}

Keywords Brain tumor classification, Convolutional Neural Networks, magnetic resonance imaging, deep learning, training accuracy, validation accuracy.

\section{INTRODUCTION}

Brain tumor classification plays a prevalent issue in medical image processing[1]. In this day and age, one reason in increase of mortality among the general population is brain tumor. It consists of group of tissues that predetermined by a slow inclusion of asymmetrical cells. A gliomas can affect the function of the brain and can be life forbidding depending on its position and measure of growth. We utilize a medical imaging system called as Magnetic resonance imaging (MRI) to entrance gliomas in clinical practice. Gliomas can be either second rate or high evaluation[3]. Brain tumors are acted towards with chemotherapy, radiation treatment or a blend of them to get recouped from brain tumors. There are various proposed calculations in ongoing examinations for highlight extraction and classification of brain tumors[4].

For the analysis of these abnormalities, various modalities are used. Among these entire image imaging modalities MRI is used as the most best and widely used for tumor diagnosis in Machine learning systems. It is difficult to find the brain

Revised Manuscript Received on February 15, 2020.

* Correspondence Author

Shravya Shetty, Department of Computer Science and Engineering, NMAM Institute of Technology, Nitte, India.

Jyothi Shetty, Department of Computer Science and Engineering, NMAM Institute of Technology, Nitte, India.

(C) The Authors. Published by Blue Eyes Intelligence Engineering and Sciences Publication (BEIESP). This is an open access article under the CC BY-NC-ND license (http://creativecommons.org/licenses/by-nc-nd/4.0/) tumors at beginning stage. Most of the brain tumors are noidentified ever after the symptoms appear[5].

MRI demonstrates the tumor in the brain. Once the tumor is found, the common way to diagnose the brain tumor type is to look all the consequences from the specimen of tissue after a Medical procedure. An MRI utilizes magnetic field to manufacture total pictures of the body and can be utilized to quantify the measure of the tumor. Deep learning adds more complexity in to the model. It is the extending family of machine learning method. The characteristic idea of Deep learning is to procure an information or ability in the portrayal of information through expanding reflection layers[6]. It has accumulated a lot of research topics in various computer science areas. The essence of Deep learning is to learn the representation of data through increasing abstraction layers. This transforms in to different function that result to data representation in a hierarchy way. Past works of deep learning based CAD framework can give and help the specialists in determination and treatment of the brain tumors. A Convolutional Neural Network (CNN) is a category of Deep Neural networks is utilized to investigate visual symbolism. In this review, we focus around the arrangement of brain tumor pictures in MRI. Classification is the way toward grouping the things as indicated by its type and pattern. In this paper, we introduced a programmed mind tumor classification strategy reliant on Convolutional Neural Networks[9].The outstanding fundamental engineering of CNN includes a Rectified Linear Unit (ReLu), sigmoid, hyperbolic tangent (Tanh), a convolution, and a pooling layer [1].

\section{RELATED WORK}

A technique called image classification is used to extract the classes of data from the multi-band raster pictures. In machine learning we make use of two important type of learning known as supervised and unsupervised learning. These two types of learning consist of different categories of algorithms. Both are most normal methodologies however article based picture examination is exceptional and most recent system as referenced than staying two and in this strategy, high goals pictures are utilized as information. The supervised classification utilizes from the earlier learning or data to decide the information structure. They incorporate ANN, SVM, k-NN. Unsupervised order is a significant strategy for programmed investigation of a few mind dementias. Unsupervised order methods, for example, K-means and FCM[1]. Classification is a successive thought in our present life and a valuable apparatus to regroup heterogeneous components into few classes containing the most homogeneous components.

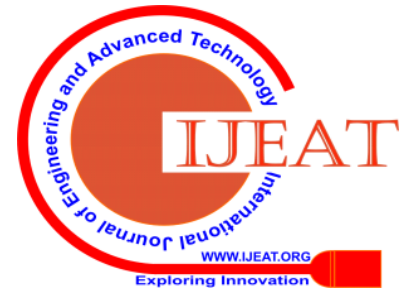




\section{Classification of Brain Tumor using Convolutional Neural Networks}

Most arrangement techniques have been connected in different settings, for example, object acknowledgment, enlistment, and division and highlight extraction, bringing about further advances to answer the requests and necessities of the clinical application space[2]. Jun Cheng et al. [3] elaborated a classification of tumor in to two phases called as offline database building and online recuperation. According to the offline database organize, the brain tumor pictures are set up in progressive advances. Various methods have been introduced such tumor division, feature extraction, and partition metric learning. The information brain picture will be arranged in like manner and difference the removed feature and the informed expelled estimations which are secured in the online database using web learning process. This technique fails to use neural framework approach but resulted in giving a classification accuracy of $94.68 \%$.

Gawande and Mendre [4] build a Deep Neural Network working on autoencoders so as to organize the mind tumor. Pereira et al. [5] likewise investigating the utilized size $3 \times 3$ portions so as to get to the more profound engineering and keep away from the overfitting. Before getting into the CNN layers, they likewise explored the utilization of power standardization as the pre-preparing venture. In this examination, we research and work on the execution of CNN on grouping a few brain tumor kinds of determination issues so as to show signs of improvement precision result. Classification arrangement results rely on the quantity of classes picked by the client. For the most part, a machine learning order strategy for classification of brain tumor consists of a large numbers of brains MRI images with recognized ground truth from various instances to prepare on. For the most part, man-made consciousness and earlier learning are joined to tackle the division issue. Presently, high division exhibitions are gotten by deep learning strategies [6].

While, in a regression, a hyper-plane ought to be found so that it fits most extreme information focuses. K-means is utilized to order protests for example pixels into k number of classes bases on same highlights. It isolates the picture in to bunch with the goal that the territories of intrigue fall in to a similar group[8].

The classification is finished by limiting the square of distances between the articles and the relating group or centroid.

\section{RESULTS AND DISCUSSIONS}

In this examination, $\mathrm{CNN}$ is acquainted based on the characterization of brain tumors. A perfect CNN design is constructed including multiple layers. CNN design is composed up of convolutional layers, pooling layer and fully connected layers trailed by one shrouded layer of 64 neurons is recognized. All convolution layers in the structures are of size $3 \times 3$.

'Adam' analyzer is used in this learning system that is considered to be a strategy for stochastic improvement. This improvement made use of stochastic angle drop standard. 'Adam' analyzer is able to adjust to new conditions. This analyzer is used in this examination due its potential advantage that helps to manage deserving points that supports on uproarious or noisy issues. The activation functions such as ReLU, sigmoid and tanh are used in this examination that can be utilized as our classification task. The activation function does the non-straight change to the information making it competent to learn and perform confused undertakings. The dimension of the maxpooling layer is $2 \times 2$ and the entire completely associated layer (called 'thick' in keras) utilizes 64 neurons. Rather than regular characterization procedures in which separated highlights are supported into frameworks, Convolutional Neural Networks (CNN) normally increase delegate composite features extracted from the given datasets.

CNN based brain tumor classification is a challenging task that mainly revolves around system based structure as opposed to the number of images. These images are divided in two group called tumor and non tumor brain images. Hence Convolutional neural network is used for automatic brain tumor classification. A CNN consists of an input layer, output layer and multiple of hidden layers. Max-pooling is a pooling layer that is used to down sample the task. This results in reducing the dimensionality of the feature map. Max pooling also result in reducing the computational cost. Pooling layer is optional. It can be skipped. The activation function used in CNN design results in transferring signals from one layer to another layer. Another layer known as flattening layer exist to vectors the dimensional matrix of feature maps. Towards the finish of a CNN, the last pooling layer goes about information and is referred as Fully Connected layer. The Fully connected layers holds composite and information data gathered from all the convolutional layers. In this examination, observed by the previous work, we explore and research the use of CNN on classifying number of brain tumor images in order to obtain a progress result.

Datasets: Web will be a wellspring of applicable information (investigate papers, proposition and books). The public available brain tumor dataset is used in this study. This dataset is provided with 1350 images belonging to 2 classes i.e. tumor and non tumor used for training phase and 606 images belonging to 2 classes used for testing phase.

In this examination, the hyperparameters are used that includes the amount and size of filters contained in the convolution layers, size of maxpooling portion, numbers of neurons used in the fully connected layers are kept stable. There is an advantage of using Convolutional neural network over other classifiers as kernels used in CNN layers have same weights for all the inputs. In normal neural network, image cannot be scalable but in CNN, images can be scalable. The approximation of loss and accuracy change as shown by the use of the structure. The four metrics are used from the training set and validation set known as accuracy and loss. The dimension of the right theories by the classifier additionally for the training set data or the validation set data is characterized as accuracy. Training loss is characterized as the error on the training data set whereas validation loss is the error after running the validation data set through the trained Model.

In the event that the precision of training set and validation set will increment for each epochs of training then the classifier model is recognized as 'good fit'. The classifier model is assessed to undergo an overfitting problem when the accuracy of validation set decreases, the accuracy of training set becomes greater.

Published By: 


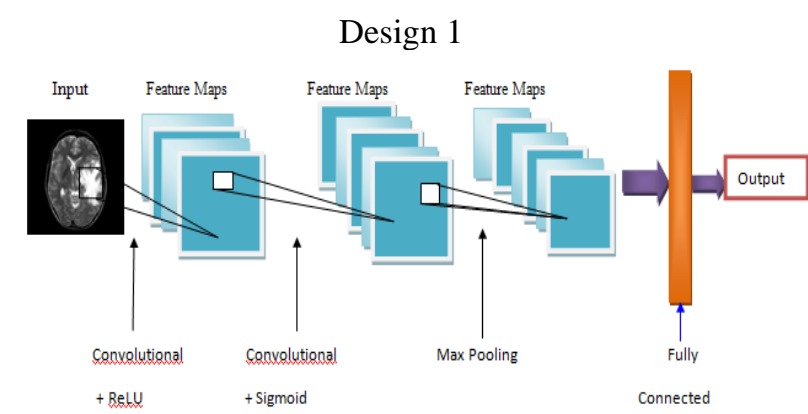

Design 3

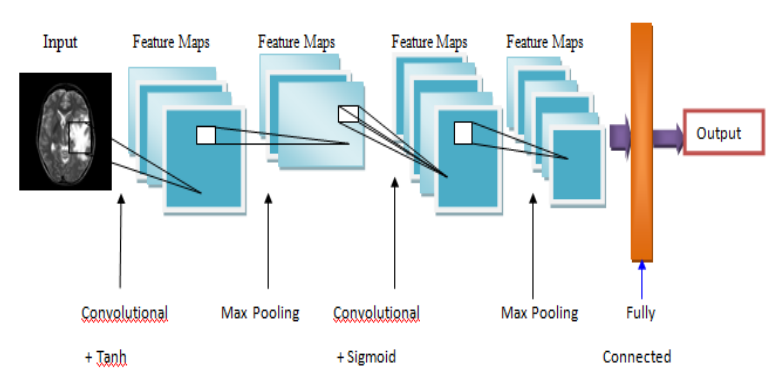

Design 5

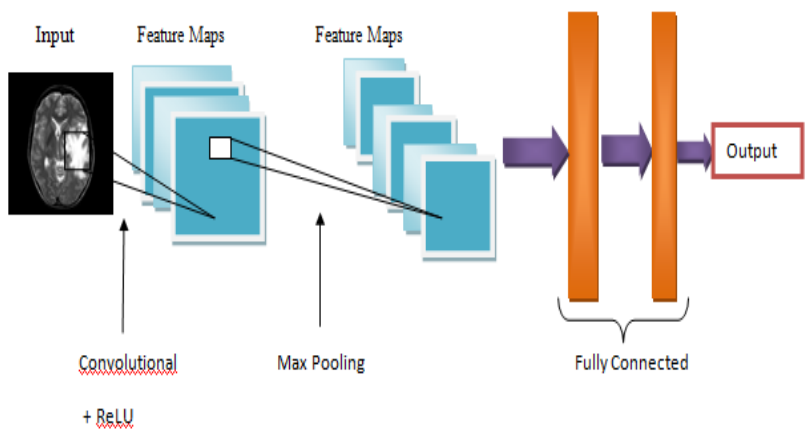

Design 2

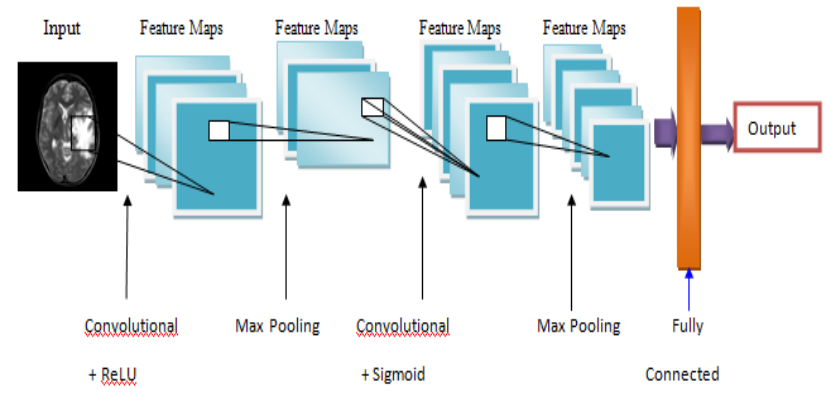

Design 4

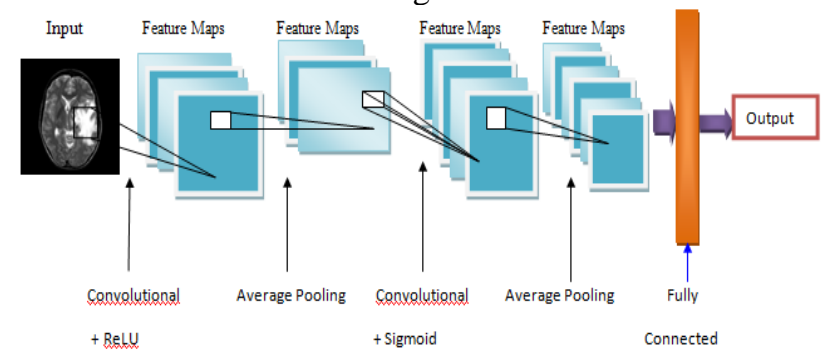

Figure 1: The different designs of CNN

Figure 1 shows the outline structure of different designs of CNN. In each design we have made changes based on layers like adding more number of layers or deleting some layers or using different activation functions. We have also made changes in design 4 using different pooling layers called average pooling layer. Activation functions such as ReLu, Sigmoid and Tanh are used. These changes result in giving best accuracy value out of 5 designs. Overfitting is caused when we train a model with lot of data. The model doesn't categorize the data correctly. Underfitting suggests a model that cannot capture the underlying trend of data. It obliterates the accuracy of the model. Underfitting can be stayed away from by utilizing more information and furthermore lessening the features by feature selection. The values of loss and accuracy for five different designs differ as stated to the performance of the design based on their layers.

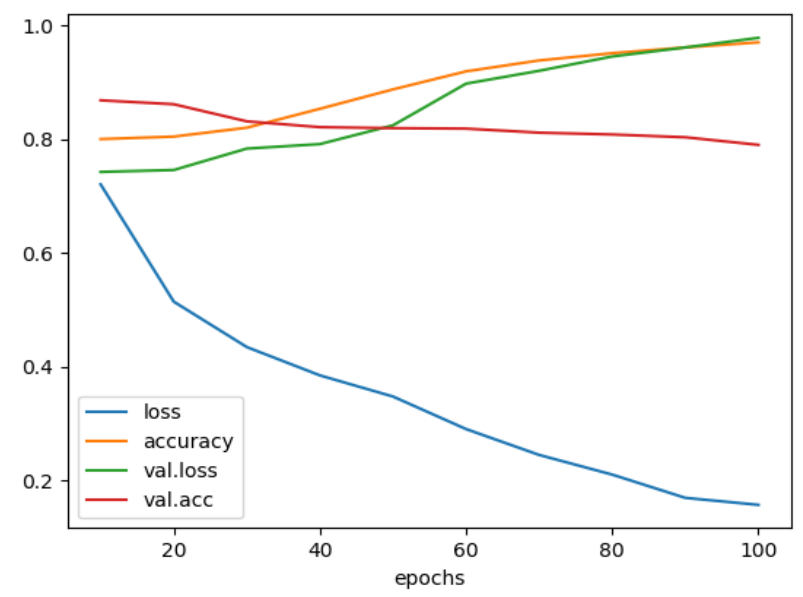

Figure 2: loss and accuracy of Design 1 


\section{Classification of Brain Tumor using Convolutional Neural Networks}

In figure 2, we have plotted the graph for the values of loss and accuracy obtained for training and validation set of design 1 . Here we use ReLu and sigmoid activation function. In this design we use one maxpooling layer.

In other designs we make use of two maxpooling layers. Removal of maxpooling layer results in increasing the dimensionality. This design achieves a training accuracy and validation accuracy of $97.02 \%$ and $80.31 \%$ at 100 epochs. In this design we make use of one max pooling layer.

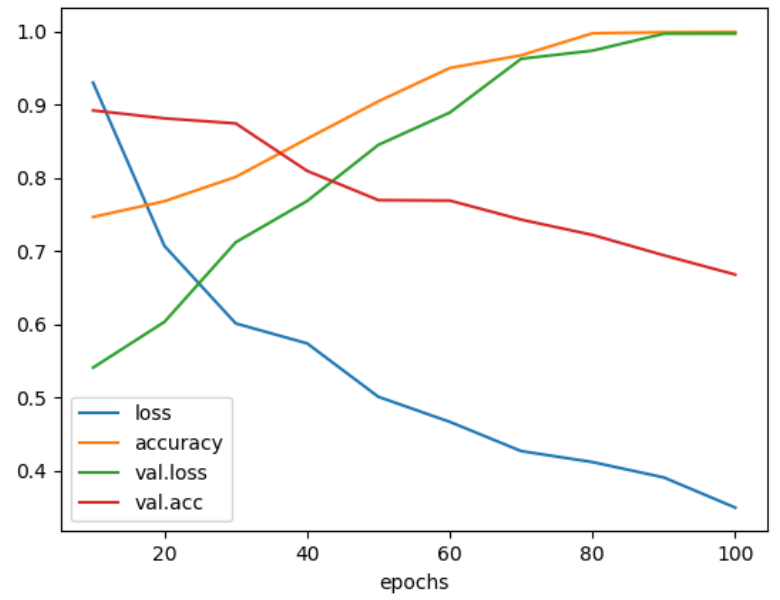

Figure 3: loss and accuracy of design 2

Figure 3 achieves a training accuracy and validation accuracy of $99.99 \%$.and $69.07 \%$ at 100 epochs. In design 2, we make use of Hyperbolic tangent (tanh) and sigmoid activation function and plot the graph for accuracy and loss.

Tanh is similar to sigmoid but better compared to it. Adding more layers helps to extract more features. This can be done up to a certain limit. It's not necessary that adding more layers result in giving more accuracy in $\mathrm{CNN}$ model. When compared to design1, we trained CNN design 2 with two layers of max pooling to increase the accuracy. It result in giving better accuracy on training data but performed very poor on validation data. This results in overfitting problem. Increase in accuracy depends upon how large dataset is being used for CNN designs.

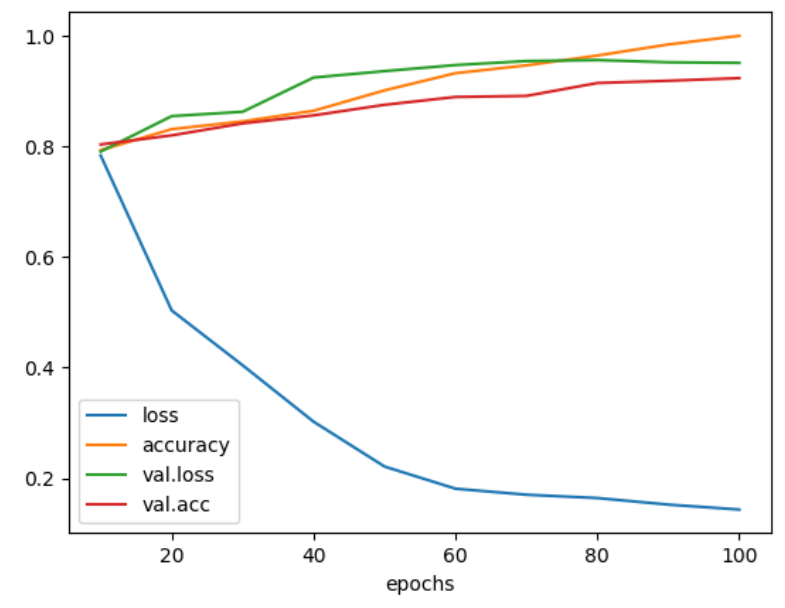

Figure 4: loss and accuracy of design 3

In design 3, we make use of activation functions i.e. Rectified Linear Unit (ReLU) and sigmoid. ReLU is one of the most common used activation functions in image classification task. It is utilized in practically all the convolutional neural networks. Sigmoid is used to predict the probability as an output. This design achieves a training accuracy and validation accuracy of $99.99 \%$ and $92.34 \%$ at 100 epochs as shown in the figure 4 . ReLU and Tanh performance depends on the design of the model, the hyperparameters and the features that we are attempting to capture.

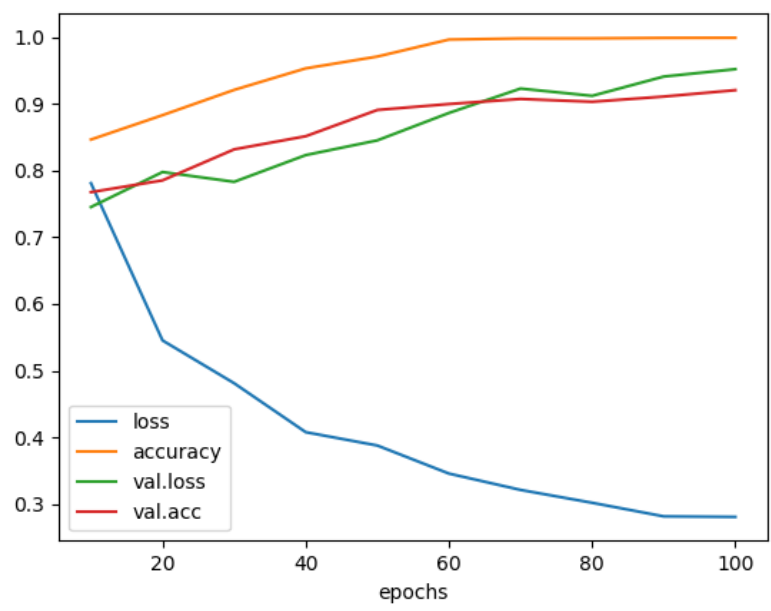

Figure 5: loss and accuracy of design 4

In design 4, we change the pooling layer from maxpooling to average pooling layer. An average pooling layer performs down-testing by partitioning the contribution to rectangular pooling portions and assessing the mean estimations of each segment. The activation function used in this design is ReLU and sigmoid function. This design achieves a training accuracy and validation accuracy of $99.99 \%$ and $88.48 \%$ at 100 epochs as shown in the figure 5.

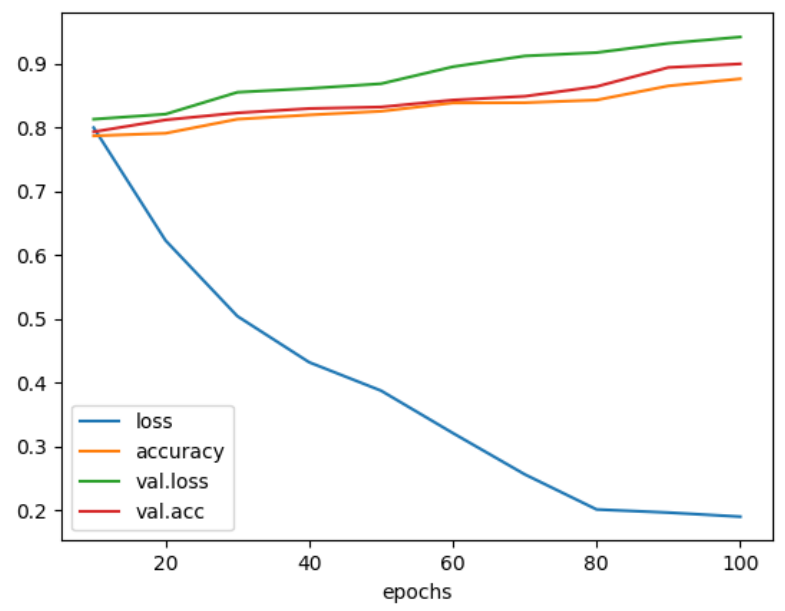

Figure 6: loss and accuracy of design 5

In figure 6, the training accuracy and validation accuracy is $87.65 \%$ and $89.99 \%$ at 100 epochs. Here we use more than one fully connected layer that helps in extracting global relationship between the features. It helps to make the input patterns easier to learn for the model.

By taking a glance at the working of 5 designs we could see that all designs misfortune demonstrates an expanding pattern regarding number of epochs in the design 3. The image is passed through a series of convolutional layers, non linear, pooling layers and fully. 
We have analyzed that CNN design with one maxpooling layer gives the training accuracy of $97.02 \%$ at 100 epochs and CNN design with two maxpooling layers gives best training accuracy of $99.99 \%$ at 100 epochs.

In design 2 and 3 we have used different activation functions. Design 3 result in giving a best training accuracy and validation accuracy of $99.99 \%$ and $92.34 \%$ at 100 epochs. This indicates that relu and sigmoid function used in design 3 gives a best accuracy when compared to the Tanh and sigmoid activation function used in design 2. ReLU is better than Tanh. Today, ReLu is the most common used activation function. It uses simple mathematical function for computation and easier to understand. When comparing the accuracy of all 5 designs, design 3 gives the best training and validation accuracy for the given datasets.

\section{CONCLUSION}

In this paper, we have constructed 5 different CNN designs that are used to categorize the brain images according to their characteristics and examine their results and find the best design based on their accuracy. We recognized an ideal CNN design comprising of convolutional layers, activation functions (ReLu, Sigmoid and Tanh), and pooling layers, trailed by one concealed layer of 64 neurons. The metrics used in this study is accuracy and loss of training and validation set. Design 3 demonstrates as the epoch's increases the training and validation accuracy increases and it contains best accuracy, hence it is considered to be the best design out of 5 designs. The training accuracy and validation accuracy of design 3 , best case scenario is $99.99 \%$ and 92.34\%, individually.

\section{REFERENCES}

1. Wu, J. (2017). Introduction to Convolutional Neural Networks. National Key Lab for Novel Software Technology Nanjing University, China.

2. Li, Miao, et al. \&quot;A review of remote sensing image classification techniques: The role of spatio-contextual information.\&quot; European Journal of Remote Sens- ing 47 (2014):389-411.

3. Cheng, J., Huang, W., Cao, S., et al. (2015). Classification via Tumor Region Augmentation and Partition. PLoS One, 10(10).

4. Gawande, S. S., Mendre, V. (2017). Brain Tumor Diagnosis Using Deep Neural Network (DNN). International Journal of Advanced Research in Electrical, Electronics and Instrumentation Engineer- ing, 5(5).

5. Pereira, S., Pinto, A., Alves, V., et al. (2016). Brain Tumor Segmentation Using Convolutional Neural Networks in MRI Images. IEEE Transactions on Medical Imaging,35(5),1240-1251.

6. Lavanyadevi, R., Machakowsalya, M., Nivehitha, J., et al. (2017), Brain tumor classification and segmentation in MRI images using PNN, IEEE Xplore.

7. Browniee, J. (2016, March 21). Overfitting and Underfitting With Machine Learning Algorithms. Retrieved from https:// machine learning mastery.com/overfitting-and-underfitting-withmachine-learning-algorithms/.

8. Varade, A. A., Ingle, K. S. (2017). Brain MRI Classification Using PNN and Segmentation Using K Means Clustering, International Journal of Advanced Research in Electrical, Elec- tronics and Instrumentation Engineering, 6(5), 6181-6188

9. . Wu, J. (2017). Introduction to Convolutional Neural Networks. National Key Lab for Novel Software Technology Nanjing University, China.

10. Siegel, R. L., Miller, K. D., Jemal, A. (2016). Cancer statistics, 2016. CA: A Cancer Journal for Clinicans, 66(1), 07-30.

11. Varade, A. A., Ingle, K. S. (2017). Brain MRI Classification Using PNN and Segmentation Using K Means Clustering, International Journal of Advanced Research in Electrical, Elec- tronics and Instrumentation Engineering, 6(5), 6181-6188.

12. Simonyan, K., Zisserman, A. (2015). Very Deep Convolution Networks for Large-Scale Image Recognition, ICLR 2015.

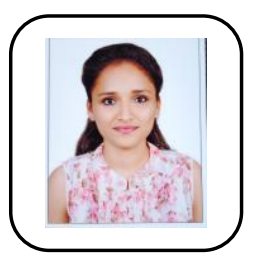

\section{AUTHORS PROFILE}

Shravya Shetty BE in Computer Science and Engineering. Pursuing M. Tech in Computer Science and Engineering at NMAMIT Nitte, Karkala. Area of interest is Deep Learning. Research work carried out Analysis of Brain Tumor Classification Using Convolutional Neural Network.

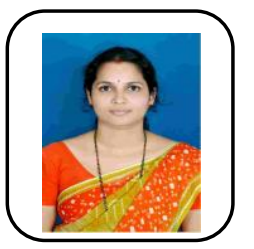

Jyothi Shetty Working as Professor in the Department of Computer Science and Engineering at NMAM Institute of Technology, Nitte, India. Her research interests include Cloud Computing, Machine Learning, Deep Learning, Optimization and Natural Language Processing. 